\title{
Two case reports and a literature review on eosinophilic fasciitis
}

\author{
Diana Mazilu1,2, Ioana Saulescu ${ }^{1,2}$, Ruxandra Ionescu ${ }^{1,2}$ \\ 1 "Carol Davila" University and Pharmacy, Bucharest, Romania \\ 2 "Sf. Maria" Clinical Hospital, Bucharest, Romania
}

\begin{abstract}
Eosinophilic fasciitis is a rare disorder of unknown etiology and poorly understood pathogenesis. It may be triggered by excessive exercise, physical factors such as radiation therapy, exposure to certain medications, infections, the initiation of hemodialysis and some other medical conditions. Skin modifications appear with collagenous thickening of the subcutaneous fascia with typical aspect of "peau d'orange" and the "groove sign". Arthritis, myalgia, myositis, neuropathies may occur. The majority of patients have peripheral blood eosinophilia. The diagnosis is confirmed with a full thickness incisional biopsy of skin and subcutaneous tissue down to the muscle surface and fascia. Systemic glucocorticoids are the mainstay of treatment, but some patients require additional immunosuppressive therapy. The long-term prognosis of this disease is unknown and in some cases the disease may releapse. We describe two patients with eosinophilic fasciitis, their evolution and complications, associating other pathologies: morphea and antiphospholipid syndrome, making the diagnosis and the treatment more challenging.
\end{abstract}

Keywords: eosinophilic fasciitis, morphea, antiphospholipid syndrome, COVID-19

\section{INTRODUCTION}

Eosinophilic fasciitis is a rare disorder of unknown etiology and poorly understood pathogenesis. It is characterized by symmetrical induration of the skin and deeper perimuscular fascial planes $(1,2)$. The hands and feet are spared. The typical skin textures are "peau d'orange" with "groove sign". Extracutaneous manifestations may appear, such as arthritis in almost $40 \%$ of patients (3), myalgia and rarely myositis (4), as well as neuropathies (such as carpal tunnel syndrome) $(3,5)$. The disease was first described by Shulman in 1974, and later was named by Rodnan in 1975. Its incidence remains unknown, with more than 250 cases reported in the literature (6). It is more frequent in males (2:1 ratio), in the sixth decade.

Possible triggers may be strenuous exercise (7), use of adulterated rapeseed oil (epidemic outbreak in Spain in 1980s) (8), hemodyalisis, Borrelia infection, exposure to medications such as statins, ramipril, heparin, phenytoin and nivolumab (9-11) and some other external factors. An important point to remember is that eosinophilic fasciitis may be associated with autoimmune diseases such as thyroid disease (12), primary biliary cirrhosis (13), systemic lupus erythematosus (14), Sjögren syndrome (15), or hematologic disorders such as aplastic anemia, thrombocytopenia, myeloproliferative disorders, lymphoma $(3,16,17)$. The most cases of eosinophilic fasciitis are not associated with any factors and are considered idiopathic.

The histopathologic changes are induces by eosinophilia and fascia infiltration. Eosinophil specific granules contain cytokines, chemokines, and growth factors (19). These cells contribute to tissue destruction by degranulation of all major granule proteins: eosinophil cationic protein (ECP), eosinophil-derived neurotoxin (EDN), eosinophil peroxidase (EPX), and major basic protein (MBP) (19). A secondary effect is the production of leukotriene, thrombocyte activating factor and the recruitment of inflammatory cells. The inflammatory infiltrate, beside eosinophils, consists of macrophages and CD8+ T 
cells exhibiting an activated cytotoxic phenotype (20). A major role plays GM-CSF. It enhances eosinophilopoiesis, induces eosinophil's cytokine secretion and promotes eosinophil survival. In patients with active disease, elevated serum levels of IL-5 and TGF- $\beta$ have been reported. Also, metalloproteinase 1 (TIMP-1) seems to be a marker of disease activity (21).

The peripheral eosinophilia is an important laboratory marker of the disease, but it does not correlate with disease severity (3). More than 50 percent of the patients have elevated biologic inflammatory markers, such as C-reactive protein and erythrocyte sedimentation rate. Moreover, polyclonal hypergammaglobulinemia may be found (22).

The diagnostic criteria (see table 1) include a deep, full skin-to-muscle biopsy and/or magnetic resonance imaging (MRI). In the early course of the disease, an infiltration with lymphocytes, plasma cells, histiocytes, and eosinophils is found. But the presence of eosinophils does not confirm the diagnosis. One of the most important features is the thickened and sclerotic fascia. These changes appear as the disease progresses (23). Thickening and inflammation also occurs within the muscle fibers (epimysium, perimysium, endomysium). The local inflammation is similar to that seen in idiopathic inflammatory myopathies (polymyositis or dermatomyositis) (24). MRI is helpful especially in patients that refuse the biopsy. The findings that confirm fascial inflammation are the increased T2 signal in the subcutaneous and deep fascia, and the enhancement of these structures on fat-suppressed T1 images after gadolinium administration $(25,26)$.

Differential diagnosis includes a variety of other conditions presenting with skin induration and tissue fibrosis. Systemic sclerosis is an autoimmune disease characterized by functional and structural vasculopathy, perivascular inflammation, fibrosis, affecting not only the skin, but also some visceral organs (pulmonary fibrosis, pulmonary arterial hypertension, renal crisis). Patients usually present with a long time Raynaud fenomena, may have digital pitting scars or active digital ulcers.

Other non-autoimmune diseases with distinct etiologies and pathologies should be ruled out. Scleroderma-like skin thickening is found in scleromyxedema, also known as mucinosis. The characteristic clinical features of this disease are the presence of waxy, yellow skin papules. These lesions are frequently found on the face, neck, hands and arms. The histological key findings are: extensive interstitial mucin deposition throughout the dermis with thickened collagen bundles and wide intercollagenous spaces, fibrocytes and inflammatory infiltrate (6). It is associated with malignancy, especially monoclonal gammopathy and amyloidosis (27).

Scleredema is a disease with diffuse skin induration without serum autoantibodies and with no evidence of inflammation on skin biopsy. It is associated with 3 conditions: poorly controlled diabetes, monoclonal gammopathies and infections (particularly streptococcal pharyngitis) (6). Typically, skin induration involves the neck, back, interscapular region, face and chest. The histology findings are thickening of the upper and lower dermis and mucin deposition between thickened collagen bundles (6). The natural progression of the disease depends on the underlying condition.

Nephrogenic systemic fibrosis is found among dialysis-dependent patients, in patients with glomerular filtration rate less than $15 \mathrm{ml} / \mathrm{min}$, or after the administration of gadolinium. This is why a renal function evaluation before a magnetic resonance imaging (MRI) with contrast is essential. The confluent fibrotic skin induration appears rapidly (over days to weeks) and is associated with lumpy-nodular plaques, pigmentary changes and flexion contractures of the extremities. The body distribution resembles systemic sclerosis distribution. It is symmetrical affecting the extremities (including the hands), the trunk and sometimes the abdomen, but the face is usually spared. The skin plagues are irregular, resembling "peau d'orange" appearance. This disease has extra cutaneous manifestations, such as peripheral neuropathy. Histologically, fibroblast proliferation is the dominant mechanism, associated with thickened collagen bundles and mucin deposition (6).

Eosinophilic fasciitis needs a prolonged treatment, but up to one third of cases may spontaneously resolve (28). Systemic glucocorticoids are the firstline therapy. Initially, a high dose cortisone regimen is recommended. The dose is reduced depending on the skin softening $(3,29)$. There are patients that need subsequent therapy, other patients may relapse. They may require immunosuppressive therapy such as methotrexate, mycophenolate or hydroxychloroquine. The data supporting the use of these agents is limited $(3,30)$. There are case reports of refractory disease treated with infliximab, rituximab, intravenous immune globulin, Psoralen plus ultraviolet (PUVA), cyclosporine A, D-penicillamine, sulfasala- 
zine, and other immunosuppressive drugs $(3,22,31$ 37). In patients with joint contractures, fasciectomy is an option for alleviating the symptoms and for regaining the range of motion (38). For patients with no criteria for severe disease (see table 2), the treatment duration may last up to 2 years. The loss of treatment response, persistent active disease, or relapse, may indicate a subsequent neoplastic process. Unfortunately, there are no randomized trials for the evaluation of the eosinophilic fasciitis treatment.

The cases described below present this rare disorder, its possible evolution and complications. There are no standard therapies, so experience usually came from small series of cases and literature reviews, making important the communication of these cases.

\section{CASE 1 PRESENTATION}

A woman in her early 50's was admitted feeling non-specifically unwell for the last year, with progressive weight loss, abdominal distension and skin changes: hyperpigmentation and diffuse induration on the thorax and abdominal area. Six months before admission, she had a thoracic and abdominal computer tomography with no pathological findings. She had a dermatologist evaluation and the skin modifications were interpreted as being secondary to a possible alimentary trigger, or allergy, or the utilization of toxic substances. Due to the skin modification in the breast areas, other medical specialists suspected breast implants, even though the patient denied any plastic surgeries. At 6 months from the first symptoms, a peripheral eosinophilia was found. The patient underwent infection disease evaluation. Parasitic infections, HIV and Borrelia infections were excluded. Hematological evaluation concluded that it was a reactive eosinophilia of unknown cause. Due to further extension of skin modifications, the dermatologist recommended a skin biopsy. The biopsy report stated: "pigmented squamous keratinized epithelium, the absence of granular layer, the atrophy of the dermal papillae, and dermal extensive fibrosis with dermal inflammatory infiltrate with the predominance of lymphocytes. The result is suggestive for systemic sclerosis". The patient was referred to the rheumatologist.

The patient came to "Sf. Maria" Clinical Hospital, Bucharest, with extensive cutaneous modifications. Chronologically, the first skin modifications appeared on breasts. The modifications expanded to the anterior thorax and abdominal area with abdominal distension, local intensive skin tension and pruritus (see figure 1). There were some small patchy areas with hyperpigmentation. Skin modifications extended and upper and lower limbs were symmetrical affected. The "groove sign", the woody and the "peau d'orange" textures were evident (see figures 3 and 4). During complete clinical examination, Raynaud phenomena was excluded. The patient did not have sclerodactyly, or digital ulcerations, or pitting scars (figure 2). The face skin was not affected, with any telangiectasia.

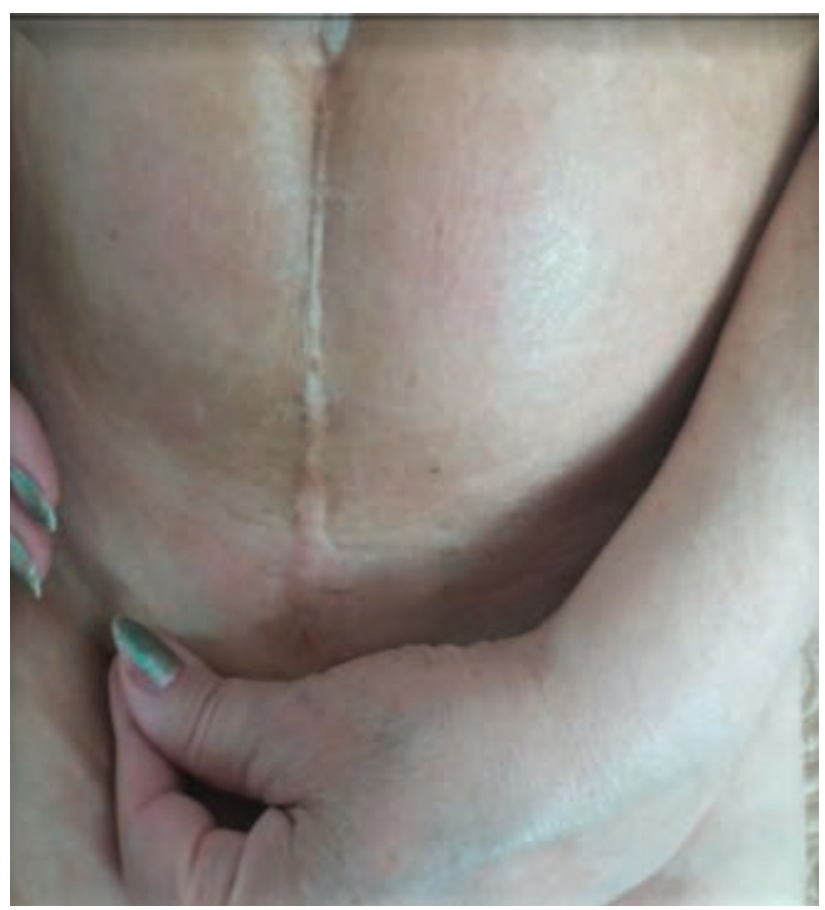

FIGURE 1. Abdominal distension. Areas with skin induration and pigmentation

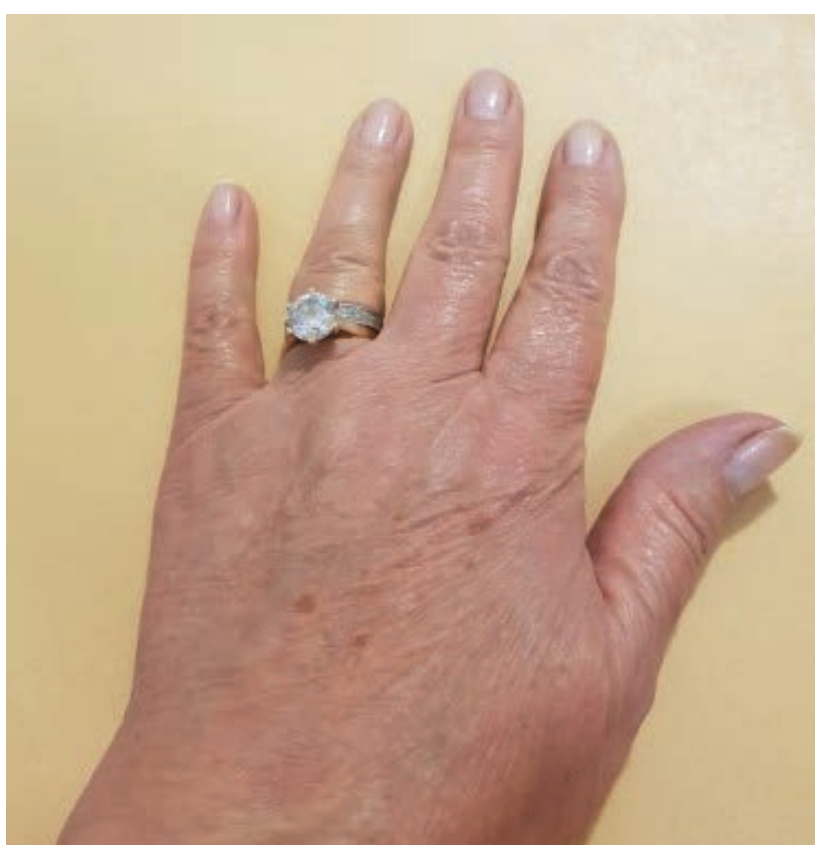

FIGURE 2. Normal skin appearance of the hands, without Raynaud phenomena, no sclerodactyly, no pitting scars, and no digital ulcerations 

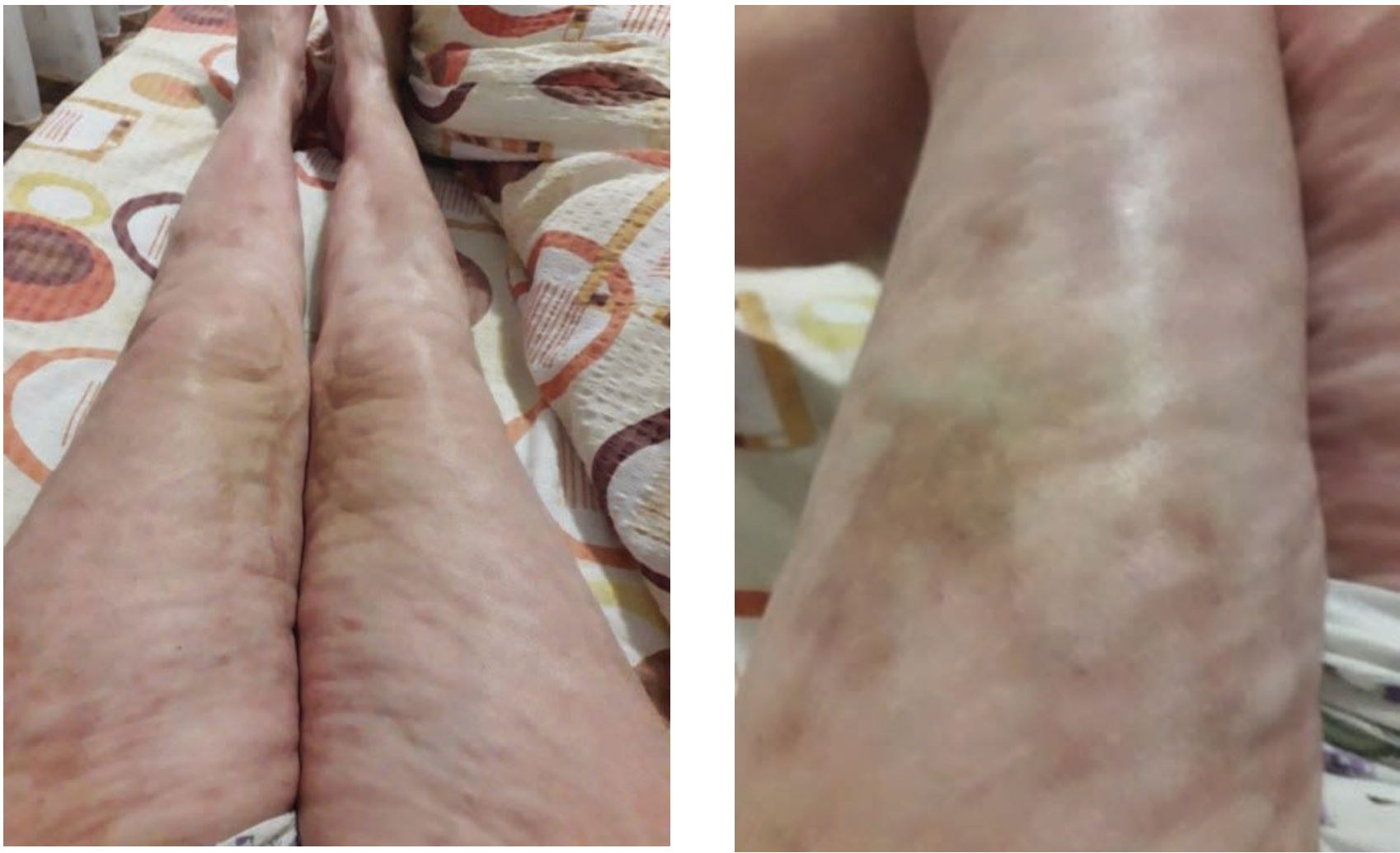

FIGURE 3 and 4. The "groove sign" and the "peau d'orange" texture

\section{Investigations}

The patient's laboratory tests revealed:

- eosinophilia $(1,400 / \mu 1,13.9 \%)$ with normal hemoglobin, leucocyte, thrombocyte levels;

- high inflammatory biomarkers (ESR $41 \mathrm{~mm} / 1$ h, CRP $39.66 \mathrm{mg} / \mathrm{l}$, fibrinogen $431 \mathrm{mg} / \mathrm{dl}$ ).

- Normal CK, CK MB, LDH, creatinine, urea, AST, ALT, GGT

To exclude autoimmune disease an anti-nuclear antibodies (ANA) panel was requested. ANA subtypes were not detected. Even though the patient did not present Raynaud phenomena, capilaroscopy was done with no modifications suggestive for connective tissue diseases. The ECG was normal and the cardiac ultrasonography revealed no significant changes. The previous biopsy was re-examined for second opinion. The second histopathologist described the fragment as a cutaneous piece with "mild atrophy of epiderma, fibrosclerosis of the derma with multiple wide septa at the level of hypoderma, an inflammatory infiltration with lympho -monocytes and eosinophils - the histological aspect suggestive for eosinophilic fasciitis".

\section{Positive diagnosis}

Taking into account the newly proposed diagnostic criteria for eosinophilic fasciitis, the diagnosis was sustained. The patient had the major criterion (symmetrical lesions on the four limbs) and a minor one (the histology features) (see table 1).

TABLE 1. Diagnostic criteria for eosinophilic fasciitis (39)

\begin{tabular}{|l|l|}
\hline \multicolumn{1}{|c|}{ Major criteria } & \multicolumn{1}{c|}{ Minor criteria } \\
\hline $\begin{array}{l}\text { Symmetrical plate-like } \\
\text { sclerotic lesions are present } \\
\text { on the four limbs. }\end{array}$ & $\begin{array}{l}\text { 1.The histology of a skin } \\
\text { biopsy that incorporates the } \\
\text { fascia shows fibrosis of the } \\
\text { subcutaneous connective } \\
\text { tissue, with thickening of the } \\
\text { fascia and cellular infiltration of } \\
\text { eosinophils and monocytes }\end{array}$ \\
\hline $\begin{array}{l}\text { However, this condition } \\
\text { lacks Raynaud's } \\
\text { phenomenon, and systemic } \\
\text { sclerosis can be excluded. }\end{array}$ & $\begin{array}{l}\text { 2. Thickening of the fascia is } \\
\text { seen using imaging tests such } \\
\text { as magnetic resonance imaging } \\
\text { (MRI). }\end{array}$ \\
\hline
\end{tabular}

A definitive diagnosis is made when a patient has the major criterion and one of the minor criteria, or the major criterion and two of the minor criteria.

\section{Differential diagnosis}

The differential diagnosis is the challenge in the disorders that present with skin induration. Due to initial limited skin modifications, localized scleroderma such as morphea and linear scleroderma were ruled out: the patient did not have a slowly progressive course typical for localized scleroderma. More than that, the patient had significant eosinophilia. Also, these data may serve as arguments for the exclusion of systemic sclerosis, beside the absence of 
Raynaud fenomena, the normal capillaroscopy, no organ involvement and the absence of ANA. A broad spectrum of scleroderma-like disease was discussed. Nephrogenic systemic fibrosis was excluded as the patient did not have an advanced renal failure and did not have administered gadolinium recently. The patient had a screening for malignancies, plasma cell disorders, monoclonal gammopathy, diabetes, scleromyxedema and scleredema were ruled out. The patient did not have an evident exposure to toxic oils, or to L-tryptophan, and did not have an organ transplant.

\section{Treatment}

Initially, the patient was treated with high dose glucocorticoids (GC). During admission, a pulse therapy with methyl prednisolone (total dose of $2 \mathrm{~g}$ ) was administered. Three days after the initiation of GC, a significant drop-down of the eosinophil count was registered: initial value of $1,400 / \mu 1$ decreased to 400 / $\mu 1$. Clinically, the patient stated a reduction in the skin tension and the pruritus especially in the abdominal area, with mild improvement in the skin induration. The patient was discharged with a medium dose of GC (prednisone $30 \mathrm{mg} /$ day). Methotrexate $(10 \mathrm{mg} /$ week) was added as an immunosuppressive and GC sparing agent. Also, she had vitamin D supplement.

The pulse therapy was administered monthly during the first 3 months after eosinophilic fasciitis was confirmed. Beside the normal value of eosinophils, a remarkable reduction in inflammatory biological markers was noticed: ESR dropped from $41 \mathrm{~mm} / 1 \mathrm{~h}$ to $21 \mathrm{~mm} / 1 \mathrm{~h}$ and CRP - from $39.66 \mathrm{mg} / \mathrm{l}$ to below 5 $\mathrm{mg} / \mathrm{l}$. During this period, prednisone dose was gradually reduced to the dose of $10 \mathrm{mg}$ prednisone per day. This was possible due to GC sparing agent - methotrexate. Methotrexate dose was increased gradually to $20 \mathrm{mg} /$ week. Patient's evolution was gratifying with reduction in the skin erythema, but most important the diminishing skin appearance of "peau d'orange" with less prominent skin "groove sign" (see table 2).

TABLE 2. Severity classification of eosinophilic fasciitis (39)

\begin{tabular}{|l|l|}
\hline Joint contracture (upper limbs) & 1 point \\
\hline Joint contracture (lower limbs) & 1 point \\
\hline Limited movement (upper limbs) & 1 point \\
\hline Limited movement (lower limbs) & 1 point \\
\hline $\begin{array}{l}\text { Expansion and worsening of skin rash } \\
\text { (progression of symptoms) }\end{array}$ & 1 point \\
\hline \multicolumn{2}{|c|}{ A total of 2 or more points is classified as severe. } \\
\hline
\end{tabular}
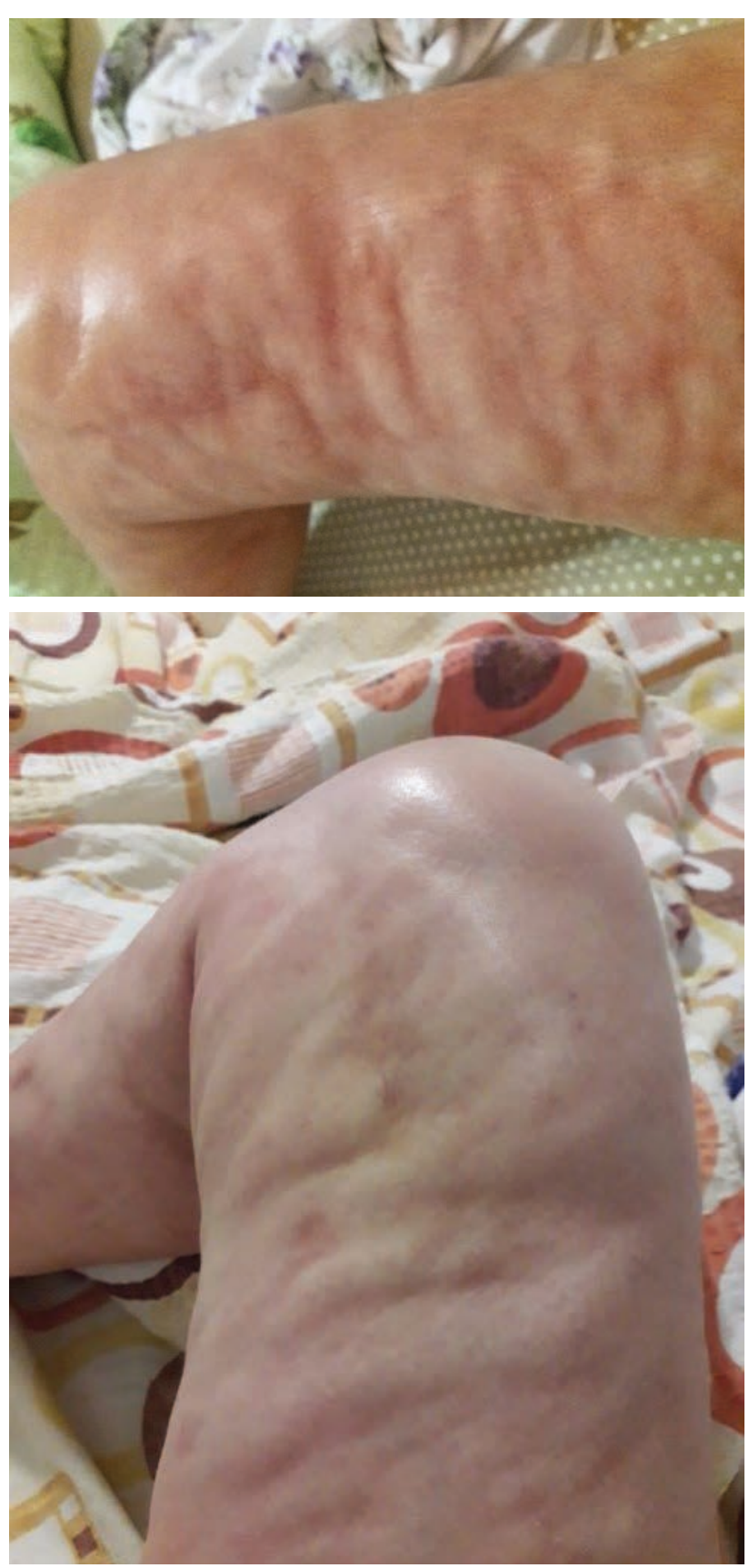

FIGURE 5 and 6. Skin modifications after 3 months of treatment. The appearance of "peau d'orange" diminished, as well as skin erythema and skin "groove sign"

\section{Outcome and follow-up}

Over the following months, the patient continued with methotrexate $20 \mathrm{mg} /$ week and vitamin D. GC therapy was slowly tapered due to the striking adverse reactions that had appeared: Cushingoid features (redistribution of body fat with buffalo hump and moon face, weight gain) and neuropsychiatric effects (emotional liability, sleep disturbance). After 2 years of immunosuppressive therapy, the patient had a favorable evolution with normal inflammatory biomarkers and normal range of the eosinophil count. She declared that skin induration was the most prom- 

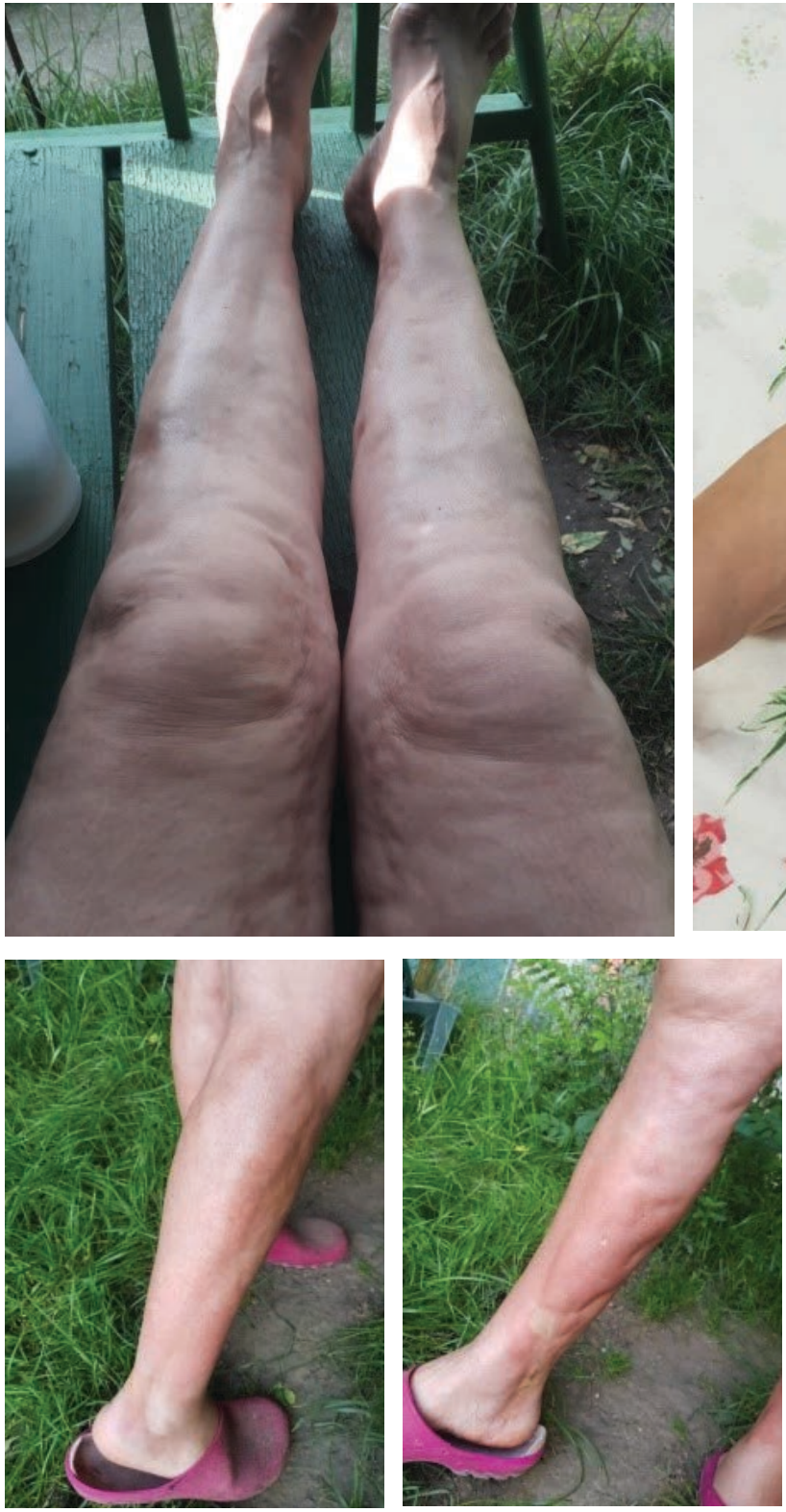

inent change that occurred during this period of treatment. Also, she noticed that all skin modifications, including the groove sign and "peau d'orange", improved in the order of their chronological appearance. The first beneficial changes were remarked in the breast skin, with local decrease in profound adherence and skin induration. Afterwards, the changes occurred in the upper limbs and lastly in the lower limbs. Even though the beneficial changes were clinically evident, new local profound adherence zones were remarked (see figures 7-10). Profound adherence and limited joint mobility were found at both ankles. Since the patient had bilateral joint contractures with limited movement, the patient was classified as having severe eosinophilic fasciitis according to the classification that was proposed in 2018 (see table 2). This particular evolution, as a disease complication, was not expected since the patient has re- sponded well to the combination of GC and methotrexate. On the other hand, it should be pointed out that GC therapy was administered for less than one year due to adverse events.

Unfortunately, the patient could not come to the clinic for the scheduled visit due to COVID-19 pandemic. A telephone call visit was done. The patient declared that she continued her immunosuppressive treatment with methotrexate $20 \mathrm{mg} / \mathrm{week}$, but new skin changes appeared. She sent us some pictures (see figures 11-12). New pigmented lesions appeared in the abdominal area and the anterior thorax. The patient said that these new lesions are painless, firm and feel like bound-down plaques. The other lesions, progressed with deep adherent bands, lesions similar to "groove sing" but they seem to be wider. Her laboratory results revealed inflammation with $\mathrm{CRP}$ value - 3 times the upper limit of normal value and eosinophilia $(10.5 \%, 851 / \mathrm{mm} 3)$. Her story will continue. She has an appointment in the clinic for evaluation.

\section{CASE 1 DISCUSSION}

Eosinophilic fasciitis is a disease of unknown etiology and pathogenesis. It may be triggered by environmental factors, and when these factors are abol- 

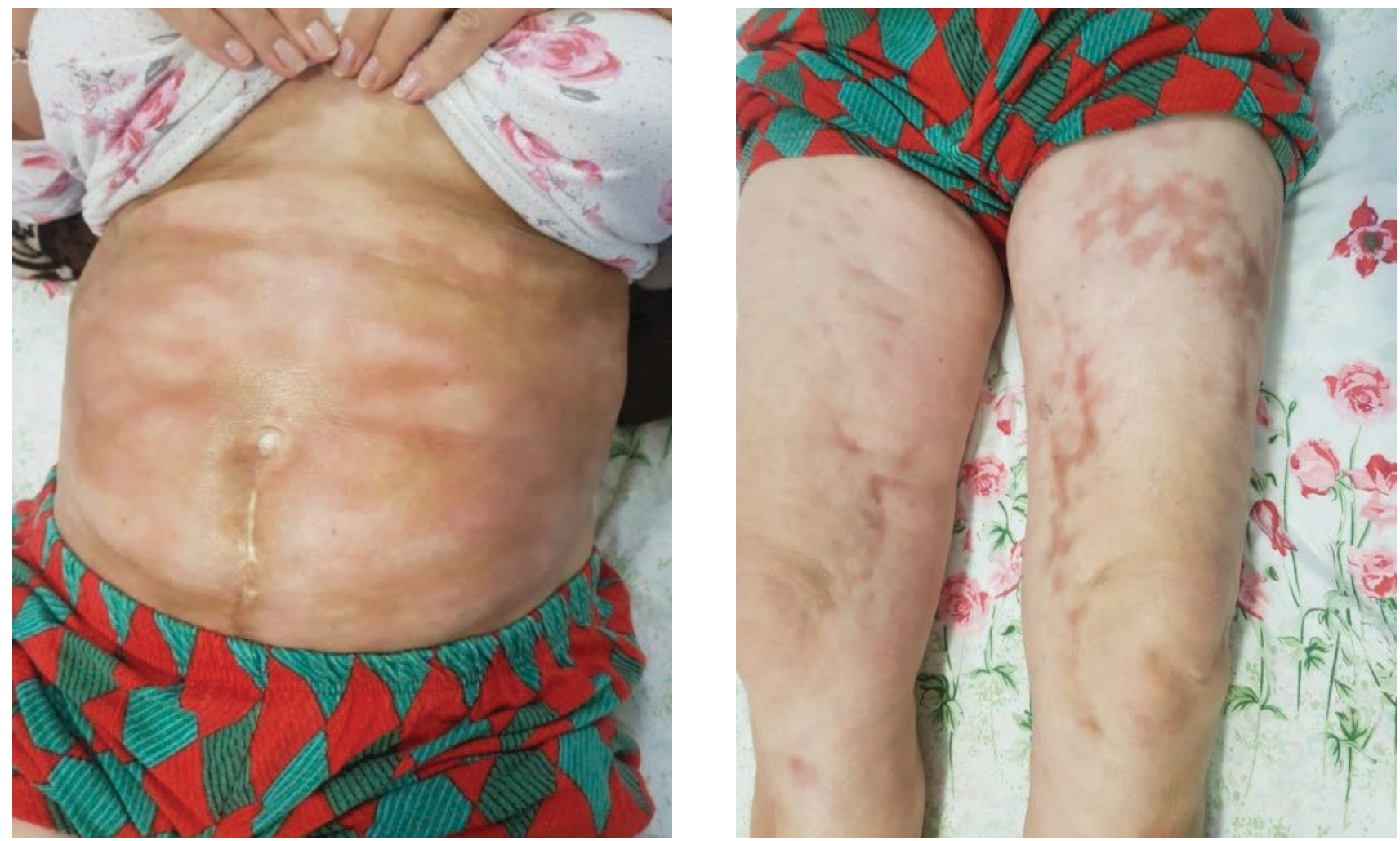

FIGURE 11 and 12. New pigmentated lesions, firm and bound-down plaques in the abdominal and anterior thorax. New profound adherent bands similar to "groove sing"

ished, the first skin manifestations such as erythema and edema, resolve in a 4 week time frame. Afterwards, the adherence diminishes and the skin softens. The natural course of this disease is the spontaneous remission. This is found within 10 to $20 \%$ of the patients (6). Unfortunately, there are patients that relapse or have complications, such as persistent fibrosis. The predisposing factors are "morphea-like" skin lesions, younger age at onset, trunk involvement and histopathological pattern with the presence of dermal fibrosclerosis (40). As it was mentioned previously, there are clinical features that contribute to severity classification (see table 2). These are joint contractures and limited movement in the upper and lower limbs, the expansion and worsening of skin rash (39).

Published data shows that in patients with extensive body surface involvement including trunk or neck involvement, GC in high doses in combination with a second immunosuppressive drug such as methotrexate or mycophenolate mofetil are a good therapeutic choice (6). This approach was used in our case, but the patient seems to have relapsed. The main predisposing factors for the relapse are GC interruption. On the other hand, the absence of response should prompt further investigation to rule out an underlying malignancy, most frequent being those hematological (6).
It should be kept in the mind that eosinophilic fasciitis may coexist with other diseases characterized by skin induration. Eosinophilic fasciitis often is regarded as part of morphea spectrum, even though the pathogenesis is different in these 2 diseases (41). The presence of thickened dermal collagen fibers is the argument for the overlap of morphea with eosinophilic fasciitis (41). In our case, the patient developed new skin modifications during immunosuppressive treatment with methotrexate. These changes are consistent with those seen in morphea. Histopathologicaly, in eosinophilic fasciitis profound fibrosis is seen. While in morphea, the skin and subcutaneous fat biopsy reveal sclerosis and fibrosis of deep reticular dermis, subcutis and rarely fascia (42). Although the initial biopsy was suggestive for eosinophilic fasciitis, the clinical progression reflects an overlap of these two diseases. There are a few cases described in the literature. Morphea lesions may appear on different parts of the body in $30 \%$ of the patients with eosinophilic fasciitis (43). The prognosis in the case of overlapping of these two disease is worse regarding skin progression comparing to each medical entity taken separately. A disease relapse requires change in treatment. The patient is waited in the clinic for further evaluation. 


\section{CASE 2 PRESENTATION}

Second case presented in this article is about a 60 -year-old man, apparently healthy until the beginning of 2020, when he developed erythema and pruritus on the extremities, followed shortly by oedema, myalgia and skin induration with limited range of motion at the big joints. Evaluation at the family doctor revealed hyper eosinophilia, absence of vasomotor manifestation or exposure to toxic substances and he was suspected of a parasitic disorder since he was working in forestry, so he was referred to a specialised department.

At that moment, clinical evaluation showed symmetrical skin induration, orange peel like skin of the extremities, but hands or feet were sparred, associated with joint contractures in the area involved (see figures 13 and 14). Routine lab tests were normal (including muscle enzyme), except for eosinophilia $(900 / \mu 1)$, polyclonal hypergammaglobulinemia and moderate inflammatory syndrome. Screening for parasitic infection was positive only for Toxoplasma gondii, but titre was high only for Ig G (approximative 3000 times higher than normal) with normal Ig $\mathrm{M}$, suggestive for chronic infection, so the specialist asked for more test. Solid neoplasia was ruled out by normal findings at the computer tomography of the thorax, abdomen and pelvis. Muscle biopsy of the lower limb was performed, and, meanwhile, other tests returned negative, like the one for chronic viral infection (Hepatitis B and C, HIV) and the one for autoimmune disorder (ANA panel).

Muscle biopsy showed preserved muscle architecture, but with some degree of atrophy of the fibres, inflammatory infiltrate with lymphocytes, plasma cells and eosinophils associated with interstitial and perivascular fibrosis.

In corroboration with the clinical findings, lab test and muscle biopsy permitted to put the final diagnosis of fasciitis with eosinophilia. He started systemic glucocorticoids with pulse-therapy $3 \mathrm{~g}$ methylprednisolone followed at home by oral therapy with equivalent prednisone dose of $0.5 \mathrm{mg} / \mathrm{kg}$ per day for 3 months with recommendation to taper the dose according to skin thickening improving.

At 3 months, patient check-up revealed normal eosinophil count, but no skin improving, also displaying now livedo reticularis. Screening for antiphospholipid syndrome was recommended and Methotrexate (MTX) was started at $10 \mathrm{mg} /$ week with the intention to lower in the next few weeks the glucocorticoid dose. Hemogram done in late 2020 au- gust, after 4 weeks of MTX, revealed low thrombocytes count $\left(80,000 \mathrm{~mm}^{3}\right)$ and since the patient did not noticed skin improvement, he stopped the MTX, remaining only on equivalent prednisone dose of near $0.5 \mathrm{mg} / \mathrm{kg}$ per day.

In September 2020, he presented at the Emergency Room of "Sf. Maria" Clinical Hospital for palpitation, dyspnoea at minimal efforts and bilateral calf pain, but more intense in the left one. He had a negative RT-PCR for SARS-CoV-2 infection. Clinical exam revealed that the patient had arrhythmia, but was hemodynamically stable with normal blood pressure, with no extra sounds on pulmonary auscultation and no need for oxygen supplementation. Extremities evaluation showed skin changes related to fasciitis with groove sign present at the superficial vein (see figure 15) accompanied by the livedo reticularis. No other clinical abnormality was found. At the end of the clinical evaluation, a diagnosis of pulmonary embolism was first suspected.

\section{Investigations}

Patient started lab and paraclinical evaluation. Electrocardiogram (EKG) showed atrial fibrillation with a medium ventricular rate (around 100/min), hemogram revealed low thrombocyte count $(45,000 /$ $\mathrm{mm}^{3}$ ), chemistry returned with high LDH (450 U) and extremely high D-dimeri level (3200), confirming that our patient had a thrombotic state. We continued the investigation with a thorax and abdominopelvic Computed Tomography and, surprisingly, there were no imaging suggestive of pulmonary embolism or parenchymal abnormality, no adenopathy, but we found acute thrombosis of left inferior cava vein in the proximal segment associated with thrombosis of the left iliac veins. Doppler ultrasounds of the vein confirmed complete thrombosis of the left femoral and popliteal vein, although clinical examination did not reveal any differences in measurements of left versus right circumference calf, probably due to skin induration related to fasciitis with eosinophilia.

\section{Differential diagnosis}

In front of our case, we had two dilemma to solve.

1. What is behind his thrombotic state?

2. Why thrombocytopenia?

Therefore, before starting anticoagulation, tests for thrombophilia and antiphospholipid syndrome were ordered. From all that, only lupus anticoagulant returned positive. An antiphospholipid syndrome diagnosis (livedo reticularis, thrombocyto- 
penia, deep vein thrombosis, positive lupus anticoagulant) was considered, remaining to repeat the test at 12 weeks apart.

About thrombocytopenia, we considered: methotrexate toxicity, but at the moment of the evaluation no other significant abnormality was found on hemogram (no leukopenia, no high mean corpuscular volume), haematological disorder, since this is found frequent in association with eosinophilic fasciitis, but peripheral smear was without any sign of this pathological condition, no lymph nodes on $\mathrm{CT}$, consume in the thrombotic state, the most probable hypothesis, since the patient was also positive for lupus anticoagulant.

\section{Positive diagnosis}

Final diagnosis was:

- eosinophilic fasciitis

- acute thrombosis of left inferior cava vein in the proximal segment associated with thrombosis of the left iliac veins, left femoral and popliteal vein

- in observation for antiphospholipid syndrome (livedo reticularis, thrombocytopenia, deep vein thrombosis, positive lupus anticoagulant in one determination)

\section{Treatment}

Facing both acute deep vein thrombosis probably related to antiphospholipid syndrome and fasciitis with eosinophilia we had two decisions to make:

1. What type of anticoagulation to choose? Or our patient was a candidate for mechanical barrier since the thrombosis was at the inferior cava vein? Since our patient had a severe thrombocytopenia (less than $50,000 \mathrm{~mm}^{3}$ ) both cardiologist and interventional cardio-vascular surgeon recommended non-invasive treatment and we started low-molecular weight heparin (LMW) followed by coumarin anticoagulant since an antiphospholipid syndrome was suspected. The thrombocytes count remained stable during the hospital surveillance.

2. What type of immunosuppressive or immunomodulatory agent to use, since our patient already tried Methotrexate with no results on the skin and there was a constant need to lower the Prednisone dose (he was more than 6 months on $0.5 \mathrm{mg} / \mathrm{kg}$ per day)? Considering both disorders, we started Hydroxychloroquine (HCQ) $5 \mathrm{mg} / \mathrm{kg}$ per day.

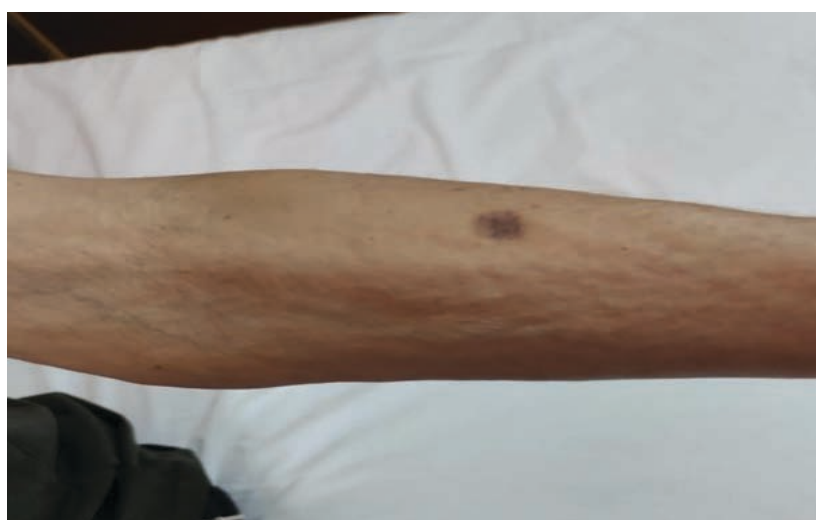

FIGURE 13. Skin induration with "orange peau" like skin

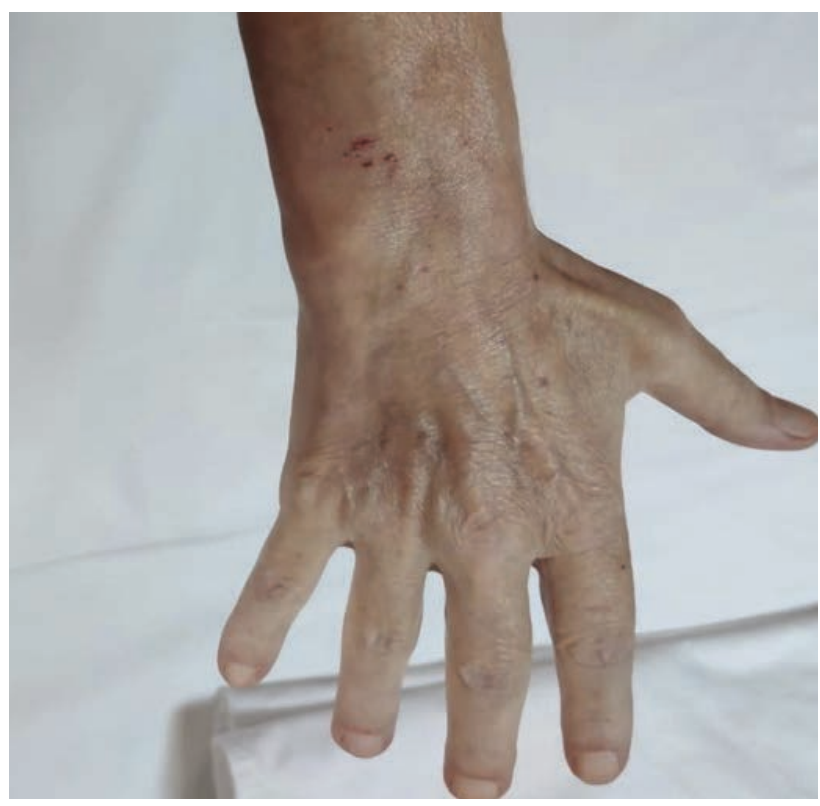

FIGURE 14. Joint contracture

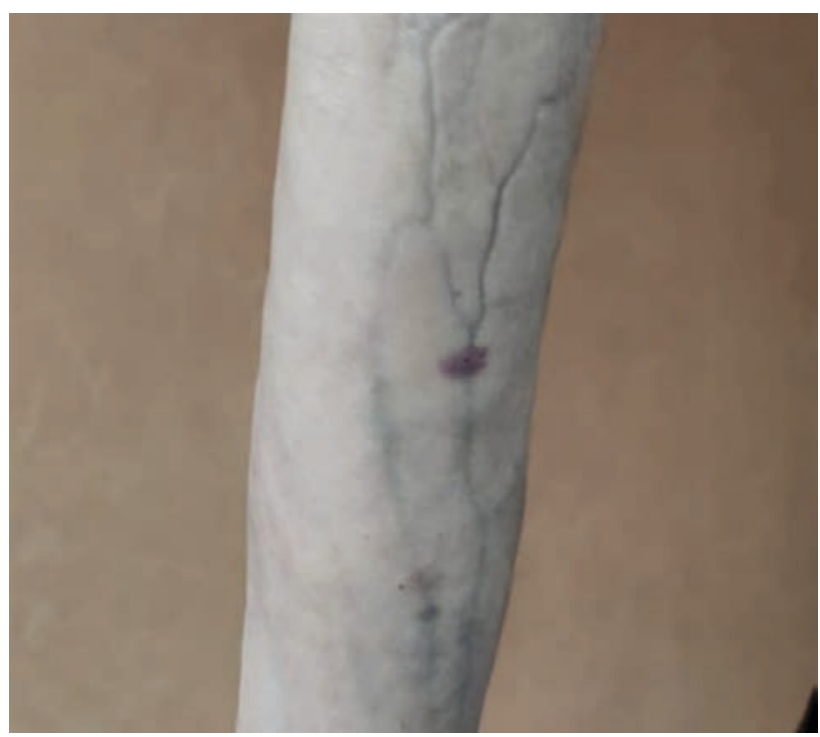

FIGURE 15. Groove sign

We also started beta-blockers for atrial fibrillation and he was converted to sinusal rhythm in 12 hours. 


\section{Outcome and follow-up}

Evolution was favourable, in one week we found sign of femoral thrombus recanalization on ultrasound, thrombocytes count did not drop during anticoagulation and the patient was ready to be discharged. During the last night in the hospital, he developed fever (38.5 ${ }^{\circ} \mathrm{C}$ ), chills, he became asthenic, so he was isolated and a new RT-PCR for SARS-COV-2 was performed. The test came back positive and the patient was transferred in a COVID-department.

He was switched to Dexamethasone and direct oral anticoagulant (DOA) - Apixaban, for an easier follow-up - and put on prophylactic antibiotherapy, continued HCQ and developed viral pneumonia with a rapid good improvement, having a negative RTPCR at 12 days after the one that was positive.

Patient returned in our clinic 1-month post-COVID. We noticed a significant skin improvement, with almost disappearing of the livedo reticularis and softening of the skin with good mechanical impact on big joints contractures. We also checked the deep vein thrombosis evolution and we found that left femoral veins were complete permeable and popliteal vein was partial permeable. Moreover, thrombocytes were around $100,000 \mathrm{~mm}^{3}$ and D-dimeri were normal. Although we rather preferred classical oral anticoagulants than DOA since we suspected an antiphospholipid syndrome, because of the good evolution we continued DOA. As per glucocorticoid intake, patient was able to gradual reduce the dose, being at a $10 \mathrm{mg}$ equivalent Prednisone per day and continued to reduce it. We asked him to return in two months for reassessment.

\section{CASE 2 DISCUSSION}

Second case is about a patient diagnosed with eosinophilic fasciitis at the beginning of the 2020, with no proven solid or hematologic neoplasia at the moment of diagnosis and no response at initial treatment represented by systemic glucocorticoids $(29,43)$. Data from the literature suggests that starting treatment early in the oedematous phase will increase the rate of response (3). Although he had a prompt diagnosis and received high dose of glucocorticoids, after 3 months no skin improvement was noticed, only eosinophil count normalised.

Many patients do not respond to initial treatment $(3,29)$ and they need immunosuppressive or immunomodulatory drugs with the purpose to improve the outcome or to lower the glucocorticoid dose. Being a rare disease, no randomised study exists in order to choose between different agents, but skin being the major organ involved, Methotrexate seems to be the most used, probably having scleroderma as example (44). Our patient received low - dose Methotrexate (10 mg / week to start) as a second line therapy, but at 4 weeks a moderate thrombocytopenia was noticed with no changes in red line cells. He had no renal insufficiency, he did not use concomitant therapy that could contribute to that (eg. trimethoprim - sulphamethoxazole) (45), but because no clinical improvement was noticed, Methotrexate was stopped, and folic acid was doubled for the next month.

In evolution patient developed a thrombotic state with deep vein thrombosis. He had also livedo reticularis, thrombocytopenia was persistent, being severe at that moment and was positive for lupus anticoagulant. A diagnose of antiphospholipid syndrome was now in line, having both clinical manifestation from the classification criteria, but also other highly suggestive for, like livedo reticularis. Moreover, at this point, aggravating thrombocytopenia seemed also related to this (46-48).

Why our patient developed antiphospholipid syndrome? Infectious disease, mostly viral, are frequent associated with antiphospholipid antibody or complete antiphospholipid syndrome. Although rare, parasitic infections can also trigger these manifestations and our patient had a very high titre of Ig G for Toxoplasma gondii $(49,50)$.

Link between persistent hypereosinophilia and thrombosis is well established (51) and recurrent thrombosis despite correct oral anticoagulation is often cited (52). This effect is due to inhibition of natural anticoagulant activity, enhance expression of tissue factor on monocytes and eosinophils, increased thromboxane release and thrombin generation $(52,53)$. Recent data showed that even transient eosinophilia can trigger a thrombotic state (52), but in our case eosinophil count was normal for a few months when thrombogenic state was noticed, prompting to look for another cause like antiphospholipid antibody.

Nevertheless, his thrombotic state faced another aggravating factor: infection with SARS-CoV-2 virus that could even trigger antiphospholipid antibody expression (53). Rapid favourable evolution of infection might be related to already started antiaggregant and anticoagulant therapies (Hydroxychloroquine and oral anticoagulant).

Along with all these thrombogenic state that seems to be found in our patient and highlights the 
importance of a thorough differential diagnosis, a clinical finding has also an important impact. Although he had deep vein thrombosis of the entire left inferior limb until inferior cava vein, clinical exam did not find any sign of thrombosis. An explanation might be the mechanical effect of tightened skin on venous system blocking the appearance of a swelling limb with a need for periodic screening of the deep vein system of the limbs by ultrasound.

\section{CONCLUSIONS}

Eosinophilic fasciitis is a rare, uncommon disorder frequently diagnosed as systemic sclerosis by mistake. Absence of sclerodactyly or vascular involvement should prompt this diagnosis in a patient Conflict of interest: none declared Financial support: none declared

\section{REFERENCES}

1. Rodnan GP, DiBartolomeo A, Medsger TA Jr. Proceedings: Eosinophilic fasciitis. Report of six cases of a newly recognized scleroderma-like syndrome. Arthritis Rheum. 1975 SepOct;18(5):525.

2. Doyle JA, Ginsburg WW. Eosinophilic fasciitis. Med Clin North Am. 1989 Sep;73(5):1157-66.

3. Lakhanpal S, Ginsburg WW, Michet CJ, Doyle JA, Moore SB. Eosinophilic fasciitis: clinical spectrum and therapeutic response in 52 cases. Semin Arthritis Rheum. 1988 May;17(4):221-31.

4. Nassonova VA, Ivanova MM, Akhnazarova VD, Oskilko TG, Bjelle A, Hofer PA, Henriksson KG, Ström T. Eosinophilic fasciitis. Review and report of six cases. Scand J Rheumatol. 1979;8(4):225-33.

5. Jones HR Jr, Beetham WP Jr, Silverman ML, Margles SW. Eosinophilic fasciitis and the carpal tunnel syndrome. J Neurol Neurosurg Psychiatry. 1986 Mar;49(3):324-7.

6. Boin F, Hummers L. Scleroderma-like fibrosing disorders. Rheum Dis Clin North Am. 2008;34 (1):199-220.

7. Shulman LE. Diffuse fasciitis with hypergammaglobulinemia and eosinophilia: a new syndrome? J Rheumatol. 1984 Oct;11(5):56970.

8. Alonso-Ruiz A, Zea-Mendoza AC, Salazar-Vallinas JM, RocamoraRipoll A, Beltrán-Gutiérrez J. Toxic oil syndrome: a syndrome with features overlapping those of various forms of scleroderma. Semin Arthritis Rheum. 1986 Feb;15(3):200-12.

9. Long H, Zhang G, Wang L, Lu Q. Eosinophilic Skin Diseases: A Comprehensive Review. Clin Rev Allergy Immunol. 2016 Apr;50 (2):189-213.

10. Le Tallec E, Lescoat A, Ballerie A, Cador B, Lena H, Ricordel C, Damien S, Lebbe C, Jego P, Belhomme N. Eosinophilic Fasciitis Triggered by Nivolumab: A Remarkable Efficacy of the mTOR Inhibitor Sirolimus. J Thorac Oncol. 2020 Feb;15 (2):e29-e30.

11. Chan KK, Magro C, Shoushtari A, Rudin C, Rotemberg V, Ross A, Lezcano C, Carrino J, Fernandez D, Postow MA, Apollo A, Lacouture ME, Bass AR. Eosinophilic Fasciitis Following Checkpoint Inhibitor Therapy: Four Cases and a Review of Literature. Oncologist. 2019 Oct 15:theoncologist.2019-0508.

12. Farrell AM, Ross JS, Bunker CB. Eosinophilic fasciitis associated with autoimmune thyroid disease and myelodysplasia treated with pulsed methylprednisolone and antihistamines. Br J Dermatol. 1999 Jun;140(6):1185-7.

13. Tahara K, Yukawa S, Shoji A, Hayashi H, Tsuboi N. Long-term remission by cyclosporine in a patient with eosinophilic fasciitis associated with primary biliary cirrhosis. Clin Rheumatol. 2008 Sep;27(9):1199-201.

14. Kitamura Y, Hatamochi A, Hamasaki Y, Ikeda H, Yamazaki S. Association between eosinophilic fasciitis and systemic lupus erythematosus. J Dermatol. 2007 Feb;34(2):150-2. with skin induration, particularly if blood eosinophilia is found. Although a good therapeutic effect is expected when treated with glucocorticoids or known immunosuppressive drugs (Methotrexate, Azathioprine, Hydroxychloroquine, etc.) many cases are marked by distinct evolution. These is emphasised by this article. Two patients followed in our clinic had associated morphea, respectively a thrombogenic state related to antiphospholipid syndrome, making the cases more challenging for diagnosis and treatment.

\section{Acknowledgement}

The authors contributed equally to the manuscript.

15. Maddison PJ. Mixed connective tissue disease, overlap syndromes, and eosinophilic fasciitis. Ann Rheum Dis. 1991 Nov;50 Suppl 4(Suppl 4):887-93.

16. Haddad H, Sundaram S, Magro C, Gergis U. Eosinophilic fasciitis as a paraneoplastic syndrome, a case report and review of the literature. Hematol Oncol Stem Cell Ther. 2014 Jun;7(2):90-2.

17. Chan LS, Hanson CA, Cooper KD. Concurrent eosinophilic fasciitis and cutaneous T-cell lymphoma. Eosinophilic fasciitis as a paraneoplastic syndrome of T-cell malignant neoplasms? Arch Dermatol. 1991 Jun;127(6):862-5.

18. Falanga V, Medsger TA Jr. Frequency, levels, and significance of blood eosinophilia in systemic sclerosis, localized scleroderma, and eosinophilic fasciitis. J Am Acad Dermatol. 1987 Oct;17(4):648-56.

19. Diny NL, Rose NR, Čiháková D. Eosinophils in Autoimmune Diseases. Front Immunol. 2017 Apr 27;8:484.

20. Toquet $C$, Hamidou M, Renaudin K, Jarry A, Foulc P, Barbarot $S$, et al. In situ immunophenotype of the inflammatory infiltrate in eosinophilic fasciitis. J Rheumatol 2003 Aug; 30 (8)1811-5.

21. Jinnin M, Ihn H, Yamane K, Asano Y, Yazawa N, Tamaki K. Serum levels of tissue inhibitor of metalloproteinase- 1 and 2 in patients with wosinophilic fasciitis. Dermatol. 2004;151 (2):407.

22. Lebeaux D, Sène D. Eosinophilic fasciitis (Shulman disease). Best Pract Res Clin Rheumatol. 2012 Aug;26(4):449-58.

23. Barnes L, Rodnan GP, Medsger TA, Short D. Eosinophilic fasciitis. A pathologic study of twenty cases. Am J Pathol. 1979 Aug;96(2):493518.

24. Huang KW, Chen XH. Pathology of eosinophilic fasciitis and its relation to polymyositis. Can J Neurol Sci. 1987 Nov;14(4):632-7.

25. Moulton SJ, Kransdorf MJ, Ginsburg WW, Abril A, Persellin S. Eosinophilic fasciitis: spectrum of MRI findings. AJR Am J Roentgenol. 2005 Mar;184(3):975-8.

26. Ronneberger M, Janka R, Schett G, Manger B. Can MRI substitute for biopsy in eosinophilic fasciitis? Ann Rheum Dis. 2009 Oct;68(10):1651-2.

27. Mori Y, Kahari VM, Varga J. Scleroderma-like cutaneous syndromes. Curr Rheumatol Rep. 2002 Apr;4(2):113-22.

28. Manzini CU, Sebastiani M, Giuggioli D, Manfredi A, Colaci $\mathrm{M}$, Cesinaro AM, Ferri C. D-penicillamine in the treatment of eosinophilic fasciitis: case reports and review of the literature. Clin Rheumatol. 2012 Jan;31(1):183-7.

29. Lebeaux D, Francès C, Barete S, Wechsler B, Dubourg O, Renoux $\mathrm{J}$, Maisonobe T, Benveniste $\mathrm{O}$, et al. Eosinophilic fasciitis (Shulman disease): new insights into the therapeutic management from a series of 34 patients. Rheumatology (Oxford). 2012 Mar;51(3):557-61.

30. Tkachenko E, Steuer AB, Lo K, LaChance A, Merola JF, Femia AN, Vleugels RA. Mycophenolate Mofetil for Eosinophilic Fasciitis: 
A Retrospective Analysis From 3 Tertiary Care Centers. JAMA Dermatol. 2020 May 1;156(5):595-597.

31. Bischoff L, Derk CT. Eosinophilic fasciitis: demographics, disease pattern and response to treatment: report of 12 cases and review of the literature. Int J Dermatol. 2008 Jan;47(1):29-35.

32. Schiener R, Behrens-Williams SC, Gottlöber P, Pillekamp H, Peter RU, Kerscher M. Eosinophilic fasciitis treated with psoralenultraviolet A bath photochemotherapy. Br J Dermatol. 2000 Apr;142(4):804-7.

33. Pimenta S, Bernardes M, Bernardo A, Brito I, Castro L, SimõesVentura F. Intravenous immune globulins to treat eosinophilic fasciitis: a case report. Joint Bone Spine. 2009 Oct;76(5):572-4.

34. Bonnotte B, Chauffert B, Caillot D, Martin F, Lorcerie B. Successful treatment with antithymocyte globulin and cyclosporin $A$ of a severe aplastic anaemia associated with an eosinophilic fasciitis. $\mathrm{Br} \mathrm{J}$ Rheumatol. 1998 Dec;37 (12):1358-9.

35. Jones AC, Doherty M. Eosinophilic fasciitis with late onset arthritis responsive to sulfasalazine. J Rheumatol. 1993 Apr;20(4):750-1.

36. Khanna D, Agrawal H, Clements PJ. Infliximab may be effective in the treatment of steroid-resistant eosinophilic fasciitis: report of three cases. Rheumatology (Oxford). 2010 Jun;49(6):1184-8.

37. Scheinberg M, Hamerschlak N, Kutner JM, Ribeiro AA, Ferreira $\mathrm{E}$, Goldenberg J, Kiss MH, Chahade WH. Rituximab in refractory autoimmune diseases: Brazilian experience with 29 patients (20022004). Clin Exp Rheumatol. 2006 Jan-Feb;24(1):65-9.

38. Suzuki G, Itoh Y, Horiuchi Y. Surgical management of eosinophilic fasciitis of the upper extremity. J Hand Surg Br. 1997 Jun;22(3):4057.

39. Jinnin M, Yamamoto T, Asano Y, Ishikawa O, Sato S, Takehara K, et al. Diagnostic criteria, severity classification and guidelines of eosinophilic fasciitis. J Dermatol. 2018;45(8):881-90.

40. Endo Y, Tamura A, Matsushima Y, Iwasaki T, Hasegawa M, Nagai Y, Ishikawa O. Eosinophilic fasciitis: report of two cases and a systematic review of the literature dealing with clinical variables that predict outcome. Clin Rheumatol. 2007 Sep;26(9):1445-51.

41. Mertens JS, Seyger MMB, Thurlings RM, Radstake TRDJ, de Jong EMGJ. Morphea and Eosinophilic Fasciitis: An Update. Am J Clin Dermatol. 2017 Aug;18(4):491-512.
42. Heidary N, Cheung W, Wang N, Kamino H, Franks AG Jr. Eosinophilic fasciitis/generalized morphea overlap. Dermatol Online J. 2009 Aug 15;15(8):2.

43. Antic M, Lautenschlager S, Itin PH. Eosinophilic fasciitis 30 years after - what do we really know? Report of 11 patients and review of the literature. Dermatology. 2006;213(2):93-101.

44. Berianu F, Cohen M, Abril A et al. Eosinophilic fasciitis: clinical characteristics and response to methotrexate. $J$ Rheum Dis. 2015;18 (1):91-8.

45. Jih $\mathrm{D}$, Werth $\mathrm{V}$. Thrombocytopenia after a single dose of methotrexate. Acad Dermatol. 1998;39:349-51.

46. oubi $\mathrm{E}, \mathrm{Krause} \mathrm{I}$, Fraser $\mathrm{A}$ et al. Livedo reticularis is a marker for predicting multisystem thrombosis in antiphospholipid syndrome. Clin Exp Rheumatol. 2005;23 (4):499.

47. Uthman I, Godeau B, Taher A, Khamashta M. The hematologic manifestations of the antiphospholipid syndrome. Blood Rev. 2008;22 (4):187.

48. Miyakis S, Lockshin Md, Atsumi T, Branch Dw, Brey RI, Cervera $\mathrm{R}$, et al. International consensus statement on an update of the classification criteria for definite antiphospholipid syndrome (APS). J Thromb Haemost. 2006 Feb 1;4 (2):295-306.

49. Mendoza-Pinto C, García-Carrasco M, Cervera R. Role of Infectious Diseases in the Antiphospholipid Syndrome (Including Its Catastrophic Variant). Curr Rheumatol Rep. 2018 Aug 20;20(10):62.

50. Tavakoli M, Roghaee S, Soheilein R et al. Antiphospholipid syndrome following toxoplasma retinochoroiditis. Ocul Immunol Inflamm. 2011;19:311-3.

51. Di Micco P, Scudiero O, Lombardo B, Lodigiani C. Idiopathic hypereosinophilia and venous thromboembolism: is there a pathophysiological or clinical link? J Blood Med. 2020;11:73-6.

52. Ames PRJ, Merashli M, Graf M, Scarpato N, Arcaro A, Gentile F. Eosinophilia and antiphospholipid antibody: double thrombogenic hits? J Thrombo Cir. 2016;2(1).

53. Ames $\mathrm{P}$, Margaglione M, Mackie S, Alves J. Eosinophilia and thrombophilia in Churg Strauss Syndrome: a clinical pathogenetic overview. Clin Appl Thromb Hemost. 2010;16:628-36. 